ИЗВЕСТИЯ АКАДЕМИИ НАУК ЭСТОНСКОИ ССР. ФИЗИКА * МАТЕМАТИКА PROCEEDINGS OF THE ACADEMY OF SCIENCES OF THE ESTONIAN SSR. PHYSICS * MATHEMATICS

$1988,37,1$

\title{
О ВОЗМОЖНОЙ ПРИЧИНЕ НЕЦЕНТРАЛЬНОСТИ ВОЗБУЖДЕННЫХ РТУТЕПОДОБНЫХ ПРИМЕСЕЙ В ЩЕЛОЧНОГАЛОИДНЫХ КРИСТАЛЛАХ
}

\author{
(Представил В. Хижняков)
}

Показано, что при определенных условиях квадратичное вибронное взаимодействие с $t_{1 u}$-модой может прнвести к нецентральности возбужденной ртутеподобной примеси одновременно с четным ян-теллеровским искажением по оси $z$ основания типа $\mathrm{NaCl}$. При сжатом октаэдре лигандов примесь сдвигается по осям второго порядка, перпендикулярным $z$, и симметрия центра понижается до $C_{2 v}$. При растянутом октаэдре примесь может выйти из узла по оси $z$ и образуется конфигурация симметрин $C_{4 v}$.

Примесные ионы с электронной конфигурацией атома ртути в щелочногалоидных кристаллах являются давними объектами подробного спектроскопического изучения, см., например, $\left[{ }^{1-4}\right]$. Оригинальность и разнообразие их люминесцентных свойств связаны с эффектом ЯнаТеллера на состояниях возбужденной $6 s 6 p$ электронной конфигурации $\left[{ }^{2-5}\right]$. При этом спектроскопические схемы связи в нерелаксированной и релаксированной решеточных конфигурациях существенно различаются $\left[{ }^{4,5}\right]$. В последнем случае сильная вибронная связь орбитально вырожденных $T$-термов примеси (ответственных за актуальные полосы свечения) с $e_{g}$-колебаниями окружения приводит к минимумам адиабатических потенциалов с центросимметричным $D_{4 h}$ искажением решетки. Спин-орбитальное взаимодействие слегка расщепляет эти минимумы. С учетом $t_{2 g}$-колебаний и более сложных вибронных взаимодействий упомянутые «тетрагональные минимумы» могут сосуществовать с «тригональными», ответственными за дополнительные полосы излучения.

Недавнее изучение спектрально-кинетических свойств люминесценции щелочногалоидных кристаллов с ртутеподобными одновалентными примесями $\left(\mathrm{In}^{+}\right)$выявило особенности, которые авторы [ $\left.{ }^{6}\right]$ относят за счет нецентросимметричного искажения конфигурации этих центров в возбужденном состоянии. Нецентральное расположение примеси заключается в данном случае в сдвиге ее из узла решетки перпендикулярно выделенной обычным $D_{4 h}$ ян-теллеровским искажением оси. Применительно к данному классу систем эффект нецентральности ранее не обсуждался.

Внецентровая стабилизация малых примесных ионов в щелочногалоидных кристаллах $\left[{ }^{7,8}\right]$ и ряде оксидов $\left[{ }^{9,10}\right]-$ хорошо известное явление. С решеточно-динамической позиции возникновению нецентральности отвечает нестабильность узлового положения примеси по нечетному колебанию трансляционного типа вследствие малости сил отталкивания примеси с окружением при узловой конфигурации $\left[{ }^{11}\right]$. Детальной причиной тому может быть малое перекрывание электронных облаков (малый ионный радиус примеси), линейное электрон-фо- 
нонное взаимодействие в условиях псевдоэффекта Яна-Теллера $\left[{ }^{12,13}\right]$ (поляризационные эффекты) или квадратичный эффект Яна-Теллера на полярном колебании $\left[{ }^{12}\right]$.

В рассматриваемой задаче нецентральность обязана, очевидно, электрон-фононному взаимодействию и в данной заметке показано, что внецентровая конфигурация типа конфигурации, указанной в [ [ $\left.{ }^{6}\right]$, может при определенных условиях возникать в результате квадратичного эффекта Яна-Теллера на $t_{1 u}$-моде. Нельзя, конечно, исключить и возможности псевдоэффекта Яна-Теллера, но это потребовало бы добавления к схеме энергетических уровней возбужденной ртутеподобной примеси четного состояния, генетически связанного с ее соседями. Аналогично $\left[{ }^{14-17}\right]$, наряду с эффектом Яна-Теллера первого порядка на $e_{g}$-колебаниях мы учтем квадратичное взаимодействие $T$-терма с комбинацией компонентов $X, Y, Z$ нечетного колебания $t_{1 u}$, имеющей симметрию $e_{g}$. В простейшем случае в $t_{1 u}$-колебаниях только сама примесь смещается из узла по осям 4-го порядка, см. [[3]. Поскольку $T \times e$-ян-теллеровская задача диагональна в пространстве компонентов $T$-терма, будем рассматривать лишь один $(z)$ из трехкратно ориентационно вырожденных листов адиабатического потенциала

$$
\begin{gathered}
F_{z}=b Q+\frac{k}{2} Q^{2}+W\left(2 Z^{2}-X^{2}-Y^{2}\right)+S Q\left(2 Z^{2}-X^{2}-Y^{2}\right)+ \\
+A\left(X^{2}+Y^{2}+Z^{2}\right)+B\left(X^{4}+Y^{4}+Z^{4}\right) .
\end{gathered}
$$

Здесь $Q$ является одним из компонентов $e_{g}$-колебаний, выбранных так, что при $Q>0$ имеет место сжатие октаэдра соседей примеси по оси $z$. Вибронная константа $W$ относится к ян-теллеровской связи второго порядка $t_{1 u}$-колебаний с примесью. Четвертый член в (1) учитывает смешивание собственно $e_{g}$-колебания с комбинацией компонентов $t_{1 u}$-колебаний той же $\left(e_{g}\right)$ симметрии. Через $k>0$ и $A>0$ обозначены упругие константы соответствующих колебаний, а $B>0-$ коэффициент ангармоничности трансляционной моды.

Обсудим сначала внецентровую конфигурацию типа указанной в $\left[{ }^{6}\right]$. Интуитивно ясно, что выход примеси в междуузельное положение перпендикулярно оси $D_{4 h}$-ян-теллеровского искажения облегчен при сжатом октаэдре $(Q>0, b<0)$, т. е. в направлениях удалившихся лигандов. Отсюда следует, что $W>0, S>0$. Потенциал (1) допускает четыре ориентационно вырожденные конфигурации симметрии $C_{2 v}$ типа

$$
\begin{array}{r}
Z_{0}=0, \quad X_{0}^{2}=Y_{0}^{2}=-\frac{1}{2 B}\left(A-W-S Q_{0}\right), \\
Q_{0}=-\left(k-\frac{S^{2}}{B}\right)^{-1}\left[b+\frac{S}{B}(A-W)\right]>0 .
\end{array}
$$

Действительность $X_{0}{ }^{2}$ и положительная определенность квадратичной формы разложения $F_{z}$ около конфигурации (2)

$$
\begin{gathered}
F_{z}\left(C_{2 v}\right)=b Q_{0}+\frac{k}{2} Q_{0}^{2}-2 B X_{0}^{4}+\frac{k}{2}\left(Q-Q_{0}\right)^{2}+\left(A+2 W+2 S Q_{0}\right) Z^{2}+ \\
+4 B X_{0}^{2}\left(X \pm\left|X_{0}\right|\right)^{2}+4 B X_{0}^{2}\left(Y \pm\left|Y_{0}\right|\right)^{2} \mp \\
\mp 2 S\left|X_{0}\right|\left(X \mp\left|X_{0}\right|\right)\left(Q-Q_{0}\right) \mp 2 S\left|X_{0}\right|\left(Y \mp\left|Y_{0}\right|\right)\left(Q-Q_{0}\right)
\end{gathered}
$$

определяют условия стабилизации этой конфигурации

$$
A-W<|b| S k^{-1}
$$


$S^{2}<B k$.

Вследствие наличия недиагональных членов в (3) возникают трй гибридные $Q-X-Y$ моды с частотами

$$
\begin{gathered}
\Omega_{1}^{2}=8 B X_{0}^{2}, \\
\Omega_{2,3}^{2}=\frac{k}{2}+4 B X_{0}^{2} \pm \sqrt{\left(\frac{k}{2}-4 B X_{0}^{2}\right)^{2}+8 S^{2} X_{0}^{2}} .
\end{gathered}
$$

В соответствии с низкой симметрией конфигураций (2) около них вырождение с колебательных мод снимается полностью.

В отсутствие взаимодействия примеси с $t_{1 u}$-модой ян-теллеровское искажение $Q_{0}\left(D_{4 h}\right)=-\frac{b}{k}$. Нетрудно убедиться, что с выходом примеси из узла оно увеличивается: $Q_{0}\left(C_{2 v}\right)>Q_{0}\left(D_{4 h}\right)$, что физически естественно. Условия (4) можно интерпретировать как требование превалирования квадратичных вибронных взаимодействий $t_{1 u}$-моды над гармонической упругостью при достаточно сильной ангармонической стабилизации ее. Отметим, что минимизирующие конфигурации типа $Z_{0}=Y_{0}=0, X_{0} \neq 0$ в данной модели не возможны, как и сосуществование минимумов $D_{4 h^{-}}$и $C_{2 v}$-симметрий.

Итак, данная модель указывает на возможность реализации в результате квадратичного вибронного взаимодействия с $t_{1 u}$-колебаниями внецентровых конфигураций возбужденной примеси, указанных в [ $\left.{ }^{6}\right]$, а с выходом примеси из узла - в направлениях осей второго порядка октаэдра, перпендикулярных $z$.

Если без учета взаимодействия с $t_{1 u}$-колебанием октаэдрическое окружение примеси растянуто по оси $z$, следует ожидать выхода примеси из узла по той же самой оси (по которой анионы удалились). В этом случае $b>0, S>0, W<0$.

Возникают две ориентационно вырожденные конфигурации симметрии $C_{4 v}$

$$
\begin{aligned}
& X_{0}=Y_{0}=0, \quad Z_{0}^{2}=-\frac{1}{2 B}\left(A+2 W+2 S Q_{0}\right), \\
& Q_{0}=-\left(k-\frac{2 S^{2}}{B}\right)^{-1}\left[b-\frac{S}{B}(A+2 W)\right]<0 .
\end{aligned}
$$

Разложение $F_{z}$ около них вида

$$
\begin{gathered}
F_{z}\left(C_{4 v}\right)=b Q_{0}+\frac{k}{2} Q_{0}^{2}-B Z_{0}^{4}+\frac{k}{2}\left(Q-Q_{0}\right)^{2}+4 B Z_{0}^{2}\left(Z \mp\left|Z_{0}\right|\right)^{2}+ \\
+\left(A-W-S Q_{0}\right)\left(X^{2}+Y^{2}\right) \pm 4 S\left|Z_{0}\right|\left(Z \mp\left|Z_{0}\right|\right)\left(Q-Q_{0}\right) .
\end{gathered}
$$

Частоты гибридных $Q-Z$-мод равны

$$
\Omega_{1,2}=\frac{k}{2}+4 B Z_{0}^{2} \pm \sqrt{\left(\frac{k}{2}-4 B Z_{0}^{2}\right)^{2}+16 S^{2} Z_{0}^{2}},
$$

а условия стабильности конфигураций (6)

$$
\begin{gathered}
A+2 W<2 S b k^{-1}, \\
2 S^{2}<B k .
\end{gathered}
$$

В согласии с более высокой симметрией внецентровой конфигурации $C_{4 v}$ частоты $X$ - и $Y$-мод в ней вырождены. Как и в предыдущем случае, $Q_{0}\left(C_{4 v}\right)>Q_{0}\left(D_{4 h}\right)$.

Данная модель содержит довольно много параметров и возможность их согласованного определения из эксперимента проблематична. 
Проверить заключение о выходе примеси из узлового положения по осям второго порядка при сжатом и по оси четвертого порядка при растянутом октаэдре можно, используя действие внешнего ориентируемого электрического поля, аналогично $\left[{ }^{6}\right]$. Таким путем можно также выяснить детальный характер направлений нецентральности, перпендикулярных $z$, в случае $C_{2 v}$-конфигураций.

\section{ЛИТЕРАТУРА}

1. Lushchik, N. E., Zazubovich, S. G. Physics of Impurity Centers in Crystals. Tallinn, «Valgus», 1972, 483-504.

2. Зазубович С. Г., Хиюняков В. В. Изв. АН СССР. Сер. физ., 49, № 10, 1874$1876(1985)$.

3. Кристофель H. Н. Теория примесных центров малых радиусов в ионных кристаллах. М., «Наука», 1974.

4. Hizhnyakov, V. V., Kristoffel, N. N. The Dynamical Jahn-Teller Effect in Localized Systems. Amsterdam-New York-Oxford, North Holland, 1984, 383-438.

5. Хижняков В. В. Препринт FI-36. Тарту, 1975 .

6. Зазубович С. Г., Нагирный В. П., Соовик Т. А. Изв. АН ЭССР. Физ. Матем., 36, № 2, 146-157 (1987).

7. Дейген М. Ф., Глинчук М. Д. УФН, 114, вып. 2, 185-211 (1974).

8. Narayanamurti, V., Pohl, R. Revs Mod. Phys., 42, № 2, 201-236 (1970).

9. Вихнин В. С., Сочава Л. С., Толпаров Ю. Н. ФТТ, 20, вып. 8, 2412-2419 (1979).

10. Вихнин В. С., Сочава Л. С. ФТТ, 21, вып. 7, 2083-2090 (1979).

11. Кристофель Н. Н., Завт Г. С. ФТТ, 9, вып. 6, 1582-1589 (1967).

12. Кристофель Н. Н. ФТТ, 21, вып. 3, 895-900 (1979).

13. Берсукер Н. Б., Полингер В. З. Вибронные взаимодействия в молекулах и кристаллах. М., «Наука», 1985.

14. Берсукер Г. И., Полингер В. З. ФТТ, 26, вып. 8, 2549-2551 (1984).

15. Кристофель Н. Н. ФТТ, 23, вып. 11, 3267-3272 (1981).

16. Kristoffel, $N$. Cooperative Phenomena. Tallinn, «Valgus», 1983, 23-28.

17. Вихнин В. С. ФТТ, 23, вып. 8, 2370-2375 (1981).

\section{N. KRISTOFFEL}

\section{ERGASTATUD ELAVHOBEDASARNASTE LISANDITE MITTETSENTRAALSUSE VOIMALIKUST POHJUSEST LEELISHALOGENIIDKRISTALLIDES}

On näidatud, et teatud tingimustel võib vibroonne ruutinteraktsioon $t_{1 u}$ vōnkemoodiga pōhjustada ergastatud elavhöbedasarnase lisandi mittetsentraalsuse samaaegselt paarse Jahn-Telleri $z$-telje suunalise moonutusega $\mathrm{NaCl}$ tüüpi alusaines. Kokkusurutud ligandidest oktaeedri korral nihkub lisand $z$-teljega ristuvaid teist järku telgi pidi ning tsentri sümmeetria alaneb $C_{2 v}$-ni. Venitatud oktaeedri korral võib lisand väljuda võresōlmest $z$-telje suunas ja tekib $C_{4 v}$ sümmeetriaga konfiguratsioon.

\section{N. KRISTOFFEL}

\section{ON THE PROBABLE ORIGIN OF THE OFF-CENTRE BEHAVIOUR OF THE EXCITED MERCURY-LIKE IMPURITIES IN ALKALI HALIDE CRYSTALS}

It is shown that under certain conditions the quadratic vibronic interaction with $t_{1 u}$ mode can lead to the off-centre position of an excited mercury-like impurity together with a simultaneous even Jahn-Teller distortion along the $z$ axis of a $\mathrm{NaCl}$ type matrix. In the case of a compressed octahedron of ligands the impurity is shifted along the second-order axes perpendicular to $z$ and the symmetry of the centre is lowered to $C_{2 v}$. In the case of an elongated octahedron the impurity can leave the on-centre position along $z$ axis and a configuration with $C_{4 v}$ symmetry arises. 